\title{
Associations Between Burnout and Practice Organization in Family Physicians
}

\author{
Jessica Creager ${ }^{1}$ \\ Anastasia J. Coutinbo, MD, MHS \\ Lars E. Peterson, MD, $\mathrm{PbD}^{3,4}$ \\ 'University of Kentucky College of Medi- \\ cine; Department of Family \& Community \\ Medicine, Lexington, Kentucky \\ ${ }^{2}$ Santa Rosa Family Medicine Residency, \\ Santa Rosa, California \\ ${ }^{3}$ American Board of Family Medicine, \\ Lexington, Kentucky \\ ${ }^{4}$ Department of Family and Commu- \\ nity Medicine, University of Kentucky, \\ Lexington, Kentucky
}

\begin{abstract}
PURPOSE Burnout has been reported to be as high as 63\% among family physicians and has negative effects on physicians, patients, and the medical system. There are likely structural causes of burnout, but little is known about the relationship between practice organization and burnout. Our objective was to study this association in family physicians.
\end{abstract}

METHODS This cross-sectional study uses secondary data supplied by practicing physicians from the 2017 American Board of Family Medicine (ABFM) Family Medicine Certification examination registration questionnaire, a mandatory component of registration, yielding a 100\% response rate. Burnout was measured as a positive response to either of 2 validated questions measuring emotional exhaustion and depersonalization. Practice environment was measured with questions on work stressors and teamwork. Logistic regression determined independent associations between burnout and individual and practice characteristics.

RESULTS Of the 1,437 physicians included, the burnout rate was $43.7 \% ; 33.7 \%$ worked in hospital-owned practices and $65.5 \%$ reported no ownership stake in their practice. Controlling for personal characteristics and practice organization, being in a hospital-owned practice (odds ratio $(\mathrm{OR})=1.68 ; 95 \% \mathrm{Cl}, 1.14-2.46$ ) and being a partial owner $(\mathrm{OR}=1.67 ; 95 \% \mathrm{Cl}, 1.13-2.46)$ were positively associated with burnout. When also controlling for practice environment, no practice organization variable remained associated with burnout.

CONCLUSION Burnout in family physicians should not be attributed solely to practice organization. No single practice type or ownership status was independently associated with burnout, which indicates that any practice can attempt to mitigate burnout.

Ann Fam Med 2019;17:502-509. https://doi.org/10.1370/afm.2448.

\section{INTRODUCTION}

$\mathrm{B}$ urnout in family physicians is estimated to be as high as $63 \%$, and there is evidence that the problem is worsening. ${ }^{1}$ The negative effects of burnout are widespread, with links to physician alcohol use $^{2}$ and suicidal ideation, ${ }^{3}$ reduced quality of patient care $^{4,5}$ and worse patient outcomes, ${ }^{5-7}$ and possible overuse of health resources due to increased referrals. ${ }^{8}$ Given the high prevalence of burnout and the serious risks, there is an urgent need to uncover the causes of burnout and identify solutions to this issue.

Although many organizational risk factors for burnout have been identified (excessive workload ${ }^{9-12}$ lack of workplace control, ${ }^{10,11,13,14}$ and misalignment of physician and organizational values $\left.{ }^{10,11,13}\right)$, there is a scarcity of research linking practice type and ownership status with physician burnout. ${ }^{15}$ These aspects of the workplace are particularly relevant considering the major decline in physicians with an ownership stake in their practice. As of 2016, less than $50 \%$ of all physicians had an ownership stake in their practice, compared to the nearly $76 \%$ with an ownership stake in 1983. Hospital employment of previously independent physicians is associated with conflicts over control of the workplace. ${ }^{16}$ When physicians
Lars E. Peterson, MD, PhD

American Board of Family Medicine 1648 McGrathiana Parkway, Suite 550 Lexington, KY 40511

lpeterson@theabfm.org 
become employees of organizations, they are less able to independently define the values of the organization, leading to possible misalignment. These practice characteristics may be linked to burnout. However, based on the current literature, it is unclear whether a practice organization type is linked with burnout or if an association is mediated through risk factors such as loss of control and misalignment of physician and organizational values. Therefore, our objective was to study the associations between burnout and practice organization and environment in family physicians.

\section{METHODS}

We used data from the 2017 American Board of Family Medicine (ABFM) Family Medicine Certification Examination registration questionnaire of practicing physicians seeking to continue board certification. ${ }^{17}$ The questionnaire is a mandatory component of examination registration and is completed 3 to 4 months prior to the examination date. The questionnaire asks about practice type and ownership, scope of practice, and practice features. Each examinee is asked a core set of questions and 1 of 5 rotating question modules. We used a module that included 9 of 10 questions from the Zero Burnout Program survey (Mini Z), which has items measuring job satisfaction, stress, and electronic health record related stress. ${ }^{13}$ The single burnout item from the Mini $Z$ was replaced on the ABFM questionnaire with 2 validated items, measuring emotional exhaustion and depersonalization, that correlate highly with the Maslach Burnout Inventory. ${ }^{18}$

Prior work has shown that respondents to each module have characteristics similar to all examinees. ${ }^{17}$ The certification examination is administered twice per year and if registered twice, the most recent responses were analyzed. Physician demographics (age, sex, and degree type) were obtained from ABFM administrative databases.

Our study assessed 2 symptoms of burnout: emotional exhaustion and depersonalization. The 2 items asked physicians how often they "feel burned out from my work" and "have become more callous toward people since I took this job." Burnout was defined as the presence of either of these once a week or more. ${ }^{1,19}$ Although originally defined as having 3 components: emotional exhaustion, depersonalization, and a reduced sense of personal achievement, ${ }^{20}$ there is evidence that a reduced sense of personal accomplishment develops independently and tends to correlate more weakly with most important causes and effects of burnout. ${ }^{21,22}$ For these reasons, it is reasonable to use only the first 2 of 3 original domains to assess burnout. $1,2,4,8,9,12,23-29$
We characterized practice type into 9 categories using 2 variables. First, we identified solo private practices by those physicians reporting solo practice size and a private practice type. Any other private practice was categorized as group private practice. We combined responses for Rural Health Clinic, Indian Health Service, and non-federal government clinic, into an "other, public" category. We kept existing response options for federally qualified health center, hospitalowned, academic health center, federal (military, Veterans Administration/Department of Defense), and health maintenance organization. Finally, we combined workplace clinic and other into a miscellaneous category. Practice ownership options included: no ownership, partial owner, sole owner, and self-employed, (independent contracting, including locums tenens).

We dichotomized the Mini $Z$ variables into yes/ no, indicating the presence of each construct. ${ }^{14}$ Individual demographic characteristics included age (categorized by decade), sex, allopathic vs osteopathic training, international medical graduate status, and identification as a core faculty member in medical education (medical school or residency). The sample was restricted to those providing primarily outpatient continuity care and excluded those without electronic health records, as dependent Mini $\mathrm{Z}$ questions were not asked of those respondents.

We calculated descriptive statistics and then tested bivariate associations between our measures and burnout using $\chi^{2}$ tests. We assessed for differences in practice environment by practice type and ownership. Finally, we used logistic regression to determine adjusted associations with burnout. We ran 3 sequential models to determine whether practice type or ownership status was associated with burnout independently of personal characteristics and practice environment. The first model included personal characteristics alone, as past work has shown female physicians aged under 40 years and over 60 years have higher odds of burnout. ${ }^{30}$ The second model added practice type and ownership, and the final model added practice environment variables.

All analyses were conducted in SAS version 9.2 (SAS Institute, Inc). This study was approved by the American Academy of Family Physicians Institutional Review Board.

\section{RESULTS}

Of the 10,106 physicians who completed the 2017 ABFM Family Medicine Certification Examination registration questionnaire, 1,510 were administered the modified Mini $\mathrm{Z}$ module, and, of these, 1,437 physicians met our inclusion criteria. As the questionnaire is 
a mandatory component of examination registration, we had a $100 \%$ response rate.

Our sample was approximately evenly divided between the sexes ( $45.7 \%$ females), and approximately one-fifth of physicians were aged 60 years or older (18.0\%) (Table 1). Just over one-third of physicians were faculty members (33.7\%). When characterized by practice type, the largest cohort of physicians worked in private practices $(34.2 \%)$, a majority of whom worked in a group practice $(24.1 \%)$. The second largest cohort worked in hospital-owned practices (33.7\%). Most physicians did not have any ownership stake in the practice $(65.6 \%)$, and those with partial ownership comprised $17.5 \%$ of our sample. A majority of physicians reported practice environments with high stress (54.6\%), satisfactory number of hours worked (69.8\%), good control over their workload (68.2\%), good value alignment with department leaders $(63.8 \%)$, and efficient teamwork $(92.8 \%)$. Less than one-half of the physicians reported sufficient time for documentation (47.4\%) and a chaotic work environment (26.7\%). The prevalence of burnout was $43.7 \%$.

Of the personal characteristics, being female or aged younger than 40 years was associated with a higher burnout rate, and being on faculty was associated with a lower burnout rate (Table 2). Practice type was substantially associated with burnout with the highest prevalence seen in federal $(51.7 \%)$ and hospital-owned $(48.4 \%)$ practices. Lower rates of burnout were seen in academic health centers (33.3\%) and solo private practices (34.9\%). Burnout was also substantially associated with practice ownership status with partial ownership having the highest burnout rate $(48.8 \%)$ and self-employment having the lowest burnout rate $(28.9 \%)$.

We found little variation in the distribution of practice environment variables in each practice type (Table $3)_{i}$ however, we did find variation in practice environment variables by physician ownership (Table 4) where self-employed physicians reported higher rates of good control over workload ( $84.4 \%$ vs $68.2 \%$ ) and sufficient time for documentation (77.8\% vs $46.4 \%)$. At the same time, self-employed physicians had lower rates of high stress $(42.2 \%$ vs $54.6 \%)$, chaos (15.6\% vs $26.7 \%)$, and excessive time spent on electronic health records at home (28.9\% vs $52.6 \%)$.

Table 5 shows the adjusted associations between burnout and our variables of interest. In the first (regression) model examining personal characteristics only, being aged 60 years or older and being a faculty member were associated with significantly reduced odds of burnout. Female sex did not have a significant independent association with burnout. In the second model, which controlled for personal characteristics as well as practice organization features, faculty status was no longer a protective factor against burnout. Regarding practice type and ownership, both working in hospital-

\section{Table 1. Characteristics of Family Physicians $(\mathrm{N}=1,437)$}

\begin{tabular}{|c|c|}
\hline Characteristics & No. (\%) \\
\hline \multicolumn{2}{|l|}{ Personal } \\
\hline \multicolumn{2}{|l|}{ Age, y } \\
\hline$<40$ & $143(10.0)$ \\
\hline $40-49$ & $552(38.4)$ \\
\hline $50-59$ & $484(33.7)$ \\
\hline$\geq 60$ & $258(18.0)$ \\
\hline Female sex & $656(45.7)$ \\
\hline US Medical Graduate & $1,124(78.2)$ \\
\hline Doctor of Osteopathy degree & $163(11.3)$ \\
\hline Faculty & $484(33.7)$ \\
\hline \multicolumn{2}{|l|}{ Practice organization } \\
\hline \multicolumn{2}{|l|}{ Practice type } \\
\hline Solo private practice & $146(10.2)$ \\
\hline Group private practice & $346(24.1)$ \\
\hline Federally qualified health center & $85(5.9)$ \\
\hline Other public & $71(4.9)$ \\
\hline Hospital-owned & $484(33.7)$ \\
\hline Academic health center & $102(7.1)$ \\
\hline Health maintenance organization & $75(5.2)$ \\
\hline Federal & $58(4.0)$ \\
\hline Miscellaneous & $70(4.9)$ \\
\hline \multicolumn{2}{|l|}{ Practice ownership } \\
\hline Self-employed & $45(3.1)$ \\
\hline Sole owner & $197(13.7)$ \\
\hline Partial owner & $252(17.5)$ \\
\hline No ownership & $943(65.6)$ \\
\hline \multicolumn{2}{|l|}{ Practice environment } \\
\hline \multicolumn{2}{|l|}{ Vulnerable patients, $\%$} \\
\hline$<10$ & $556(38.7)$ \\
\hline $10-49$ & $583(40.6)$ \\
\hline$\geq 50$ & $298(20.7)$ \\
\hline $\begin{array}{l}\text { I feel a great deal of stress because of my job } \\
\text { (agree/strongly agree) }\end{array}$ & $785(54.6)$ \\
\hline $\begin{array}{l}\text { My control over my workload is (satisfactory/ } \\
\text { good/optimal) }\end{array}$ & $980(68.2)$ \\
\hline $\begin{array}{l}\text { Sufficiency of time for documentation is } \\
\text { (satisfactory/good/optimal) }\end{array}$ & $667(46.4)$ \\
\hline $\begin{array}{l}\text { Which number best describes the atmosphere } \\
\text { in your primary work area? ( } 4 \text { or } 5 ; 5=\text { hec- } \\
\text { tic, chaotic) }\end{array}$ & $384(26.7)$ \\
\hline $\begin{array}{l}\text { My professional values are well aligned with } \\
\text { those of my department leaders (agree/ } \\
\text { strongly agree) }\end{array}$ & $917(63.8)$ \\
\hline $\begin{array}{l}\text { The degree to which my care team works effi- } \\
\text { ciently together is (satisfactory/good/optimal) }\end{array}$ & $1,333(92.8)$ \\
\hline $\begin{array}{l}\text { The amount of time I spend on the EHR at } \\
\text { home is (excessive/moderately high) }\end{array}$ & $756(52.6)$ \\
\hline $\begin{array}{l}\text { Satisfaction with hours worked (very satisfied/ } \\
\text { satisfied) }\end{array}$ & $1,003(69.8)$ \\
\hline $\begin{array}{l}\text { Burnout (symptoms of emotional exhaustion } \\
\text { or callousness) }\end{array}$ & $628(43.7)$ \\
\hline
\end{tabular}


owned practices (odds ration $(\mathrm{OR})=1.68 ; 95 \% \mathrm{CI}$, 1.14-2.46) and partial practice ownership $(\mathrm{OR}=1.67$; $95 \% \mathrm{CI}, 1.13-2.46)$ were associated with burnout. The

\section{Table 2. Characteristics of Family Physicians by Burnout Status $(\mathrm{N}=1,437)$}

\begin{tabular}{|c|c|c|c|}
\hline Characteristics & $\begin{array}{c}\text { Burned Out } \\
\text { (n=628), } \\
\text { No. }(\%)\end{array}$ & $\begin{array}{c}\text { Not Burned Out } \\
(n=809), \\
\text { No. }(\%)\end{array}$ & $\begin{array}{c}P \\
\text { Value }\end{array}$ \\
\hline \multicolumn{4}{|l|}{ Personal } \\
\hline Age, y & & & .0008 \\
\hline$<40$ & $68(47.6)$ & $75(52.5)$ & \\
\hline $40-49$ & $264(47.8)$ & $288(52.2)$ & \\
\hline $50-59$ & $211(43.6)$ & $273(56.4)$ & \\
\hline$\geq 60$ & $85(33.0)$ & $173(67.1)$ & \\
\hline Female sex & $309(47.1)$ & $347(52.9)$ & .017 \\
\hline us Medical graduate & $496(44.1)$ & $628(55.9)$ & .54 \\
\hline Doctor of Osteopathy degree & $64(39.3)$ & $99(60.7)$ & .23 \\
\hline Faculty & $193(39.9)$ & $291(60.1)$ & .037 \\
\hline \multicolumn{4}{|l|}{ Practice organization } \\
\hline Practice type & & & .055 \\
\hline Solo private practice & $51(34.9)$ & $95(65.1)$ & \\
\hline Group private practice & $149(43.1)$ & $197(56.9)$ & \\
\hline Federally qualified health center & $35(41.2)$ & $50(58.8)$ & \\
\hline Other public & $30(42.3)$ & $41(57.8)$ & \\
\hline Hospital-owned & $234(48.4)$ & $250(51.7)$ & \\
\hline Academic health center & $34(33.3)$ & $68(66.7)$ & \\
\hline Health maintenance organization & $34(45.3)$ & $41(54.7)$ & \\
\hline Federal & $30(51.7)$ & $28(48.3)$ & \\
\hline Miscellaneous & $31(44.3)$ & $39(55.7)$ & \\
\hline Practice ownership & & & .025 \\
\hline Self-employed & $13(28.9)$ & $32(71.1)$ & \\
\hline Sole owner & $75(38.1)$ & $122(61.9)$ & \\
\hline Partial owner & $123(48.8)$ & $129(51.2)$ & \\
\hline No ownership & $417(44.2)$ & $526(55.8)$ & \\
\hline \multicolumn{4}{|l|}{ Practice environment } \\
\hline Vulnerable patients, $\%$ & & & .082 \\
\hline$<10$ & $233(41.9)$ & $323(58.1)$ & \\
\hline $10-49$ & $275(47.2)$ & $308(52.8)$ & \\
\hline$\geq 50$ & $120(40.3)$ & $178(59.7)$ & \\
\hline $\begin{array}{l}\text { I feel a great deal of stress because of } \\
\text { my job (agree/strongly agree) }\end{array}$ & $491(62.6)$ & $294(37.5)$ & $<.0001$ \\
\hline $\begin{array}{l}\text { My control over my workload is } \\
\text { (satisfactory/good/optimal) }\end{array}$ & $318(32.5)$ & $662(67.6)$ & $<.0001$ \\
\hline $\begin{array}{l}\text { Sufficiency of time for documentation } \\
\text { is (satisfactory/good/optimal) }\end{array}$ & $184(27.6)$ & $483(72.4)$ & $<.0001$ \\
\hline $\begin{array}{l}\text { Which number best describes the } \\
\text { atmosphere in your primary work } \\
\text { area? ( } 4 \text { or } 5 ; 5=\text { hectic, chaotic) }\end{array}$ & $244(63.5)$ & $140(36.5)$ & $<.0001$ \\
\hline $\begin{array}{l}\text { My professional values are well aligned } \\
\text { with those of my department leaders } \\
\text { (agree/strongly agree) }\end{array}$ & $315(34.4)$ & $602(65.7)$ & $<.0001$ \\
\hline $\begin{array}{l}\text { The degree to which my care team } \\
\text { works efficiently together is } \\
\text { (satisfactory/good/optimal) }\end{array}$ & $563(42.2)$ & $770(57.8)$ & $<.0001$ \\
\hline $\begin{array}{l}\text { The amount of time I spend on the EHR } \\
\text { at home is (excessive/moderately high) }\end{array}$ & $393(52.0)$ & $363(48.0)$ & $<.0001$ \\
\hline $\begin{array}{l}\text { Satisfaction with hours worked (very } \\
\text { satisfied/satisfied) }\end{array}$ & $368(36.7)$ & $635(63.3)$ & $<.0001$ \\
\hline
\end{tabular}

final model controlled for personal, practice organization, and practice environment characteristics. In this model, all previous personal or practice organization variables lost their significance except for age of 60 years or older $(\mathrm{OR}=0.54 ; 95 \% \mathrm{CI}, 0.37-0.78)$.

Practice environment features associated with reduced odds of burnout were satisfaction with hours worked $(\mathrm{OR}=0.73 ; 95 \% \mathrm{CI}, 0.55$ $0.97)$, good control over workload $(\mathrm{OR}=0.58 ; 95 \% \mathrm{CI}, 0.43-0.80)$, good value alignment with department leaders $(\mathrm{OR}=0.48 ; 95 \% \mathrm{CI}$, $0.36-0.62$ ), and sufficient time for documentation $(\mathrm{OR}=0.64 ; 95 \%$ CI, 0.47-0.86). High professional stress was associated with significantly increased odds of burnout $(\mathrm{OR}=3.95 ; 95 \% \mathrm{CI}, 3.02-5.16)$.

\section{DISCUSSION}

In this study of family physicians, we found that neither practice type nor ownership were associated with self-reported burnout when controlling for other personal and practice environment risk factors. Rather, for all practice types, a practice environment that increases physician stress with poor control over workload, conflicting values with leadership, and arduous documentation burdens predicted burnout. Our findings suggest that burnout can occur in any practice type, and no specific organizational structure dooms physicians to burnout. This also suggests a common path forward: that all practices can make changes and reduce burnout.

Although our study did not show any significant association between burnout and practice type when controlling for personal and practice environment characteristics, federal and hospital-owned practices had higher prevalence of burnout overall, and therefore could be a good target for focused interventions. Burnout in these settings may be due to a complex interaction of personal and prac- 
Table 3. Practice Environment of Family Physicians by Practice Type $(\mathrm{N}=1,437)$

\begin{tabular}{|c|c|c|c|c|c|c|c|c|c|c|}
\hline $\begin{array}{l}\text { Environment } \\
\text { Characteristic }\end{array}$ & $\begin{array}{c}\text { Solo } \\
\text { Private } \\
\text { Practice, } \\
\%\end{array}$ & $\begin{array}{c}\text { Group } \\
\text { Private } \\
\text { Practice, } \\
\%\end{array}$ & $\begin{array}{c}\mathrm{FQHC}, \\
\%\end{array}$ & $\begin{array}{l}\text { Other } \\
\text { Public, } \\
\%\end{array}$ & $\begin{array}{l}\text { Hospital } \\
\text { Owned, } \\
\%\end{array}$ & $\begin{array}{l}\text { Academic } \\
\text { Health } \\
\text { Center, } \\
\%\end{array}$ & $\begin{array}{l}\text { HMO, } \\
\%\end{array}$ & $\begin{array}{c}\text { Federal, } \\
\%\end{array}$ & $\begin{array}{c}\text { Misc, } \\
\%\end{array}$ & $\begin{array}{c}\text { Total, } \\
\%\end{array}$ \\
\hline $\begin{array}{l}\text { I feel a great deal of } \\
\text { stress because of my } \\
\text { job (agree/strongly } \\
\text { agree) }\end{array}$ & 50.0 & 56.0 & 52.9 & 54.9 & 56.8 & 47.0 & 56.0 & 62.0 & 47.1 & 54.6 \\
\hline $\begin{array}{l}\text { My control over } \\
\text { my workload is } \\
\text { (satisfactoryl } \\
\text { good/optimal) }\end{array}$ & 74.6 & 76.9 & 57.7 & 70.4 & 64.3 & 63.7 & 58.7 & 60.3 & 72.9 & 68.2 \\
\hline $\begin{array}{l}\text { Sufficiency of time for } \\
\text { documentation is } \\
\text { (satisfactory/good/ } \\
\text { optimal) }\end{array}$ & 54.8 & 50.0 & 36.5 & 53.5 & 40.5 & 44.1 & 42.7 & 50.0 & 61.4 & 46.4 \\
\hline $\begin{array}{l}\text { Which number best } \\
\text { describes the atmo- } \\
\text { sphere in your primary } \\
\text { work area? ( } 4 \text { or } 5 ; \\
5=\text { hectic, chaotic) }\end{array}$ & 17.1 & 23.4 & 34.1 & 29.6 & 26.7 & 31.4 & 45.3 & 32.8 & 20.0 & 26.7 \\
\hline $\begin{array}{l}\text { My professional values } \\
\text { are well aligned with } \\
\text { those of my depart- } \\
\text { ment leaders (agree/ } \\
\text { strongly agree) }\end{array}$ & 58.2 & 69.1 & 69.4 & 64.8 & 61.6 & 69.6 & 60.0 & 55.2 & 60.0 & 63.8 \\
\hline $\begin{array}{l}\text { The degree to which } \\
\text { my care team works } \\
\text { efficiently together } \\
\text { is (satisfactory/good/ } \\
\text { optimal) }\end{array}$ & 96.6 & 96.5 & 92.9 & 90.1 & 89.5 & 88.2 & 97.3 & 93.1 & 92.9 & 92.8 \\
\hline $\begin{array}{l}\text { The amount of time I } \\
\text { spend on the EHR at } \\
\text { home is (excessive/ } \\
\text { moderately high) }\end{array}$ & 45.9 & 54.3 & 42.4 & 43.7 & 59.7 & 46.1 & 54.7 & 34.5 & 34.3 & 52.6 \\
\hline $\begin{array}{l}\text { Satisfaction with hours } \\
\text { worked (very satisfied/ } \\
\text { satisfied) }\end{array}$ & 70.6 & 72.0 & 70.6 & 71.8 & 66.7 & 71.6 & 58.7 & 69.0 & 85.7 & 69.8 \\
\hline $\begin{array}{l}\text { Vulnerable patients } \\
\quad<10 \%\end{array}$ & 51.4 & 52.3 & 2.4 & 4.2 & 38.4 & 20.6 & 33.3 & 56.9 & 42.9 & 38.7 \\
\hline $\begin{array}{l}\text { Vulnerable patients } \\
\text { 10-49\% }\end{array}$ & 30.1 & 37.6 & 10.6 & 43.7 & 52.1 & 37.3 & 49.3 & 31.0 & 34.3 & 40.6 \\
\hline $\begin{array}{l}\text { Vulnerable patients } \\
\geq 50 \%\end{array}$ & 18.5 & 10.1 & 87.1 & 52.1 & 9.5 & 42.2 & 17.3 & 12.1 & 22.9 & 20.7 \\
\hline
\end{tabular}

Table 4. Practice Environment of Family Physicians by Practice Ownership $(\mathrm{N}=1,437)$

\begin{tabular}{|c|c|c|c|c|c|}
\hline Environment Characteristic & $\begin{array}{l}\text { No Ownership, } \\
\%\end{array}$ & $\begin{array}{c}\text { Partial } \\
\text { Owner, \% }\end{array}$ & $\underset{\%}{\text { Self-Employed, }}$ & $\begin{array}{c}\text { Sole } \\
\text { Owner, \% }\end{array}$ & $\begin{array}{l}\text { Total, } \\
\%\end{array}$ \\
\hline $\begin{array}{l}\text { I feel a great deal of stress because of my job (agree/ } \\
\text { strongly agree) }\end{array}$ & 53.8 & 60.7 & 42.2 & 53.8 & 54.6 \\
\hline My control over my workload is (satisfactory/good/optimal) & 64.9 & 71.0 & 84.4 & 76.7 & 68.2 \\
\hline $\begin{array}{l}\text { Sufficiency of time for documentation is (satisfactory/good/ } \\
\text { optimal) }\end{array}$ & 44.0 & 44.8 & 77.8 & 52.8 & 46.4 \\
\hline $\begin{array}{l}\text { Which number best describes the atmosphere in your pri- } \\
\text { mary work area? ( } 4 \text { or } 5 ; 5=\text { hectic, chaotic) }\end{array}$ & 28.0 & 31.0 & 15.6 & 17.8 & 26.7 \\
\hline $\begin{array}{l}\text { My professional values are well aligned with those of } \\
\text { my department leaders (agree/strongly agree) }\end{array}$ & 63.8 & 67.9 & 64.4 & 58.4 & 63.8 \\
\hline $\begin{array}{l}\text { The degree to which my care team works efficiently } \\
\text { together is (satisfactory/good/optimal) }\end{array}$ & 91.1 & 96.4 & 91.1 & 96.5 & 92.8 \\
\hline $\begin{array}{l}\text { The amount of time I spend on the EHR at home is (exces- } \\
\text { sive/moderately high) }\end{array}$ & 52.5 & 61.1 & 28.9 & 47.7 & 52.6 \\
\hline Satisfaction with hours worked (very satisfied/satisfied) & 70.3 & 67.5 & 73.3 & 69.5 & 69.8 \\
\hline Vulnerable patients $<10 \%$ & 33.3 & 51.2 & 33.3 & 49.8 & 38.7 \\
\hline Vulnerable patients $10-49 \%$ & 42.1 & 43.3 & 37.8 & 30.5 & 19.8 \\
\hline Vulnerable patients $\geq 50 \%$ & 24.6 & 5.6 & 28.9 & 19.8 & 20.7 \\
\hline
\end{tabular}


Table 5. Adjusted Associations of Family Physicians With Burnout $(\mathrm{N}=1,437)$

\begin{tabular}{|c|c|c|c|}
\hline Characteristics & $\begin{array}{c}\text { Model } 1 \\
\text { Personal } \\
\text { OR }(95 \% \mathrm{Cl})\end{array}$ & $\begin{array}{l}\text { Model } 2 \\
\text { Personal and } \\
\quad \text { Practice } \\
\text { OR }(95 \% \mathrm{Cl})\end{array}$ & $\begin{array}{c}\text { Model } 3 \\
\text { Personal, Practice, } \\
\text { and Environment } \\
\text { OR }(95 \% \mathrm{Cl})\end{array}$ \\
\hline \multicolumn{4}{|l|}{ Personal } \\
\hline \multicolumn{4}{|l|}{ Age, y } \\
\hline$<40$ & $0.99(0.68-1.43)$ & $0.99(0.68-1.45)$ & $0.98(0.63-1.51)$ \\
\hline $40-49$ & Reference & Reference & Reference \\
\hline $50-59$ & $0.85(0.66-1.08)$ & $0.87(0.68-1.12)$ & $0.76(0.57-1.02)$ \\
\hline$\geq 60$ & $0.56(0.40-0.77)$ & $0.57(0.41-0.79)$ & $0.54(0.37-0.78)$ \\
\hline Female sex & $1.14(0.92-1.42)$ & $1.20(0.96-1.51)$ & $1.07(0.82-1.39)$ \\
\hline US medical graduate & $1.20(0.92-1.56)$ & $1.16(0.89-1.52)$ & $0.84(0.61-1.14)$ \\
\hline Doctor of Osteopathy degree & $0.74(0.53-1.05)$ & $0.71(0.50-1.01)$ & $0.77(0.52-1.14)$ \\
\hline Faculty & $0.78(0.62-0.98)$ & $0.82(0.64-1.05)$ & $0.91(0.68-1.21)$ \\
\hline \multicolumn{4}{|l|}{ Practice organization } \\
\hline \multicolumn{4}{|l|}{ Practice type } \\
\hline Solo private practice & $\ldots$ & $0.69(0.39-1.21)$ & $0.72(0.38-1.35)$ \\
\hline Group private practice & $\ldots$ & Reference & Reference \\
\hline Federally qualified health center & $\ldots$ & $1.29(0.73-2.27)$ & $1.02(0.50-2.05)$ \\
\hline Other public & $\ldots$ & $1.30(0.73-2.31)$ & $1.09(0.55-2.13)$ \\
\hline Hospital-owned & $\ldots$ & $1.68(1.1-2.46)$ & $1.22(0.78-1.89)$ \\
\hline Academic health center & $\ldots$ & $0.96(0.54-1.70)$ & $0.75(0.39-1.47)$ \\
\hline Health maintenance organization & $\ldots$ & $1.12(0.67-1.89)$ & $0.74(0.40-1.39)$ \\
\hline Federal & $\ldots$ & $1.78(0.95-3.32)$ & $1.21(0.59-2.51)$ \\
\hline Miscellaneous & $\ldots$ & $1.34(0.75-2.38)$ & $1.46(0.75-2.86)$ \\
\hline \multicolumn{4}{|l|}{ Practice ownership } \\
\hline Self-employed & $\ldots$ & $0.58(0.29-1.14)$ & $0.70(0.3-1.53)$ \\
\hline Sole owner & $\ldots$ & $1.50(0.85-2.65)$ & $1.12(0.59-2.14)$ \\
\hline Partial owner & $\ldots$ & $1.67(1.13-2.46)$ & $1.32(0.84-2.06)$ \\
\hline No ownership & $\ldots$ & Reference & Reference \\
\hline \multicolumn{4}{|l|}{ Practice environment } \\
\hline \multicolumn{4}{|l|}{ Vulnerable patients, $\%$} \\
\hline$<10$ & $\ldots$ & $\ldots$ & Reference \\
\hline $10-49$ & $\ldots$ & $\ldots$ & $1.14(0.86-1.51)$ \\
\hline$\geq 50$ & $\ldots$ & $\ldots$ & $0.93(0.62-1.38)$ \\
\hline $\begin{array}{l}\text { I feel a great deal of stress because } \\
\text { of my job (agree/strongly agree) }\end{array}$ & $\ldots$ & $\ldots$ & $3.95(3.02-5.16)$ \\
\hline $\begin{array}{l}\text { My control over my workload is } \\
\text { (satisfactory/good/optimal) }\end{array}$ & $\ldots$ & $\ldots$ & $0.58(0.43-0.80)$ \\
\hline $\begin{array}{l}\text { Sufficiency of time for documenta- } \\
\text { tion is (satisfactory/good/optimal) }\end{array}$ & $\ldots$ & $\ldots$ & $0.64(0.47-0.86)$ \\
\hline $\begin{array}{l}\text { Which number best describes the } \\
\text { atmosphere in your primary work } \\
\text { area? ( } 4 \text { or } 5 ; 5=\text { hectic, chaotic) }\end{array}$ & $\ldots$ & $\cdots$ & $1.37(1.02-1.86)$ \\
\hline $\begin{array}{l}\text { My professional values are well } \\
\text { aligned with those of my } \\
\text { department leaders (agree/ } \\
\text { strongly agree) }\end{array}$ & $\ldots$ & $\ldots$ & $0.48(0.36-0.62)$ \\
\hline $\begin{array}{l}\text { The degree to which my care team } \\
\text { works efficiently together is } \\
\text { (Satisfactory/Good/Optimal) }\end{array}$ & $\ldots$ & $\ldots$ & $1.29(0.78-2.15)$ \\
\hline $\begin{array}{l}\text { The amount of time I spend on } \\
\text { the EHR at home is (excessive/ } \\
\text { moderately high) }\end{array}$ & $\ldots$ & $\ldots$ & $1.16(0.89-1.52)$ \\
\hline $\begin{array}{l}\text { Satisfaction with Hours Worked } \\
\text { (very satisfied/satisfied) }\end{array}$ & $\ldots$ & $\ldots$ & $0.73(0.55-0.97)$ \\
\hline
\end{tabular}

tice environment features for which we did not have data. For example, our measures of practice environment were not exhaustive and we lacked data on staff turnover and engagement, which are associated with burnout. ${ }^{31,32}$ One possible mechanism for hospitalowned and federal practices to have higher rates of burnout may be from lack of clinician control over support staff, which could lead to poor team function and lengthy times to hire new clinicians

Practice ownership was similarly associated with burnout in bivariate correlations, but lost significance when controlling for other characteristics. Selfemployment, which was associated with lower levels of burnout, was also associated with a lower prevalence of many practice environment risk factors, especially insufficient time for documentation and excessive home electronic health record use. The lack of these risk factors may explain the lower rate of burnout seen in self-employed physicians and emphasizes the importance of addressing documentation burdens.

The features of a practice environment associated with burnout found in this study overlap with previous studies of family physicians. ${ }^{14}$ Our study strengthens the evidence that loss of control and insufficient time for documentation contribute to physician burnout and need to be resolved as part of the burnout solution. One proposed answer to rekindling a joy in practice is redistributing clerical burdens and workload from physicians to clinical support staff. Physicians 
in multiple types of practice settings have reported greater satisfaction with work-life balance when their practice utilizes standing orders, team meetings, and in-visit scribes, for example. ${ }^{33,34}$ One possible drawback would be that as workload for physicians is reduced, the workload for clinical support staff members may increase. By redistributing tasks, burnout may merely be shifted, rather than genuinely resolved. The prevalence of burnout in clinical support staff is already high with almost one-third of nurses reporting emotional exhaustion. ${ }^{35,36}$ The solutions laid out to increase joy in practice for physicians warrant further exploration of their effects on other members of the clinical care team before they are implemented.

Poor alignment of physicians and departmental values was also associated with burnout, consistent with results from previous studies. ${ }^{10,11,13,14}$ Our study showed that only $63.6 \%$ of physicians felt as though their values aligned well with those of their department leaders. This issue has been addressed in a non-clinical context by a collaborative rewriting of and renewed investment in the organization's mission statement, ${ }^{27}$ a relatively simple task compared to the reorganization of workloads. Considering the commonness of the issue and its association with burnout, pursuing solutions to the misalignment of values may serve as a good jumping off point for organizational leaders.

Our finding of $43.7 \%$ burnout in family physicians is substantially less than the $63.0 \%$ reported by Shanafelt et al, who used high emotional exhaustion and depersonalization scores of the Maslach Burnout

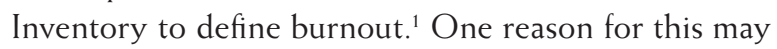
be that Shanafelt et al had a $19.2 \%$ response rate compared to our $100 \%$ response rate. Additionally, the Maslach Burnout Inventory includes a series of questions to define the presence of emotional exhaustion or depersonalization, whereas our survey only had a single item for each domain, however, the questions we used have shown good correlation with the Maslach Burnout Inventory in a comparison study. ${ }^{18}$ Another study using data from the 2016 ABFM Family Medicine Certification Examination registration questionnaire (also 100\% response rate) estimated the prevalence of burnout was $25 \% .{ }^{14}$ This study used a different question that only correlates with emotional exhaustion, thus excluding those who experienced depersonalization alone. Given these nuances, our study, with its high response rate and inclusion of both components of burnout, may better represent the prevalence of burnout in family physicians than previous studies.

Single items measuring personal accomplishment domain are not available, but requiring scores from all 3 domains has not been common, or substantiated by, the literature on physician burnout. ${ }^{37}$ If we had used the full Maslach Burnout Inventory our estimate of burnout might differ slightly.

Our study found no independent association between sex and burnout. This contrasts the paper by Weidner et al ${ }_{1}^{19}$ which found that female sex was associated with higher burnout rates. This discrepancy could be due to the differences between our study populations. The study by Weidner et al was limited to physicians at an early career stage, whereas our study included physicians at all career stages. This might indicate that sex has a greater effect on burnout at earlier career stages, but that the effect dissipates over time. This would be a good subject for future studies, especially considering the increased risk of burnout in female physicians overall.

Our study is subject to several limitations. It is cross-sectional and therefore we can only infer association, not causation. Also, our data come from a single specialty: family medicine. However, the stress of outpatient care is likely similar for most primary care physicians, allowing our results to be generalized across multiple specialties. Our data is also limited to physicians seeking continuing certification, and therefore does not represent family physicians who are not certified by the ABFM. Several of the practice types that we explored had smaller sample sizes, limiting our ability to assess their significance and increasing our risk of having a biased sample. This is counterbalanced by our survey's $100 \%$ response rate, which reduces bias. Although our study controlled for many personal and practice characteristics, we did not control for workload directly (ie, number of patient hours or number of patient encounters). We did use physician satisfaction with their work hours to try to represent subjective workload, but this may have been insufficient, as patient load could be unsatisfactory even when work hours were satisfactory. Workload has been shown by previous studies to affect burnout, $, 12,13$ and may have correlated more strongly with some practice types than others. Future work should further explore this association.

Overall, our study indicates that burnout is not intrinsic to any specific practice type or ownership status. Instead, features of the practice environment predict burnout and may provide a universal lever to lower burnout in all types of practice organizations. Future interventional studies might focus on federal and hospital-owned practices as those most in need of solving and preventing physician burnout.

To read or post commentaries in response to this article, see it online at http://www.AnnFamMed.org/content/17/6/502.

Submitted October 26, 2018; submitted, revised, March 22, 2019; accepted April 12, 2019. 
Key words: burnout; family physicians; health care organizations

Acknowledgments: Bo Fang, PhD, contributed the analysis of the data for this article.

Funding support: Funding for Ms Creager was provided by the Division of Community Medicine's Summer Research Program.

\section{References}

1. Shanafelt TD, Hasan O, Dyrbye LN, et al. Changes in burnout and satisfaction with work-life balance in physicians and the general US working population between 2011 and 2014. Mayo Clin Proc. 2015 90(12):1600-1613.

2. Oreskovich MR, Kaups KL, Balch CM, et al. Prevalence of alcohol use disorders among American surgeons. Arch Surg. 2012;147(2): 168-174.

3. Shanafelt TD, Balch CM, Dyrbye L, et al. Special report: suicidal ideation among American surgeons. Arch Surg. 2011;146(1):54-62.

4. Shanafelt TD, Bradley KA, Wipf JE, Back AL. Burnout and selfreported patient care in an internal medicine residency program. Ann Intern Med. 2002;136(5):358-367.

5. Panagioti M, Geraghty K, Johnson J, et al. Association between physician burnout and patient safety, professionalism, and patient satisfaction: a systematic review and meta-analysis. JAMA Intern Med. 2018;178(10):1317-1330.

6. Halbesleben JRB, Rathert C. Linking physician burnout and patient outcomes: exploring the dyadic relationship between physicians and patients. Health Care Manage Rev. 2008;33(1):29-39.

7. Welp A, Meier LL, Manser T. Emotional exhaustion and workload predict clinician-rated and objective patient safety. Front Psychol. 2015;5:1573.

8. Kushnir T, Greenberg D, Madjar N, Hadari I, Yermiahu Y, Bachner YG. Is burnout associated with referral rates among primary care physicians in community clinics? Fam Pract. 2014;31(1):44-50.

9. Dyrbye LN, Shanafelt TD, Balch CM, Satele D, Sloan J, Freischlag J. Relationship between work-home conflicts and burnout among American surgeons: a comparison by sex. Arch Surg. 2011;146(2): 211-217.

10. Leiter MP, Maslach C. Areas of Worklife: A structured approach to organizational predictors of job burnout. Res Occup Stress Well Being. 2003;3:91-134

11. Linzer M, Manwell LB, Williams ES, et al. Working conditions in primaryc care: physician reactions and care quality. Ann Intern Med. 2009;151(1):28-36, W6-9.

12. Shanafelt TD, Balch CM, Bechamps GJ, et al. Burnout and career satisfaction among American surgeons. Ann Surg. 2009;250(3): 463-471.

13. Linzer M, Poplau S, Babbott S, et al. Worklife and wellness in academic general internal medicine: results from a national survey. J Gen Intern Med. 2016;31(9):1004-1010.

14. Rassolian M, Peterson LE, Fang $B$, et al. Workplace factors associated with burnout of family physicians. JAMA Intern Med. 2017; 177(7):1036-1038.

15. Dyrbye LN, Shanafelt TD, Sinsky CA, et al. Burnout among health care professionals: a call to explore and address this underrecognized threat to safe, high-quality care. Natl Acad Med Perspect. 2017: 1-11. https://nam.edu/wp-content/uploads/2017/07/Burnout-AmongHealth-Care-Professionals-A-Call-to-Explore-and-Address-ThisUnderrecognized-Threat.pdf.

16. Hill JE, Wild J. Survey provides data on practice acquisition activity. Healthc Financ Manage. 1995;49(9):54-55, 58, 60 passim.

17. Peterson LE, Fang B, Phillips RL Jr, Avant R, Puffer JC. The American Board of Family Medicine's data collection method for tracking their specialty. J Am Board Fam Med. 2019;32(1):89-95.
18. West CP, Dyrbye LN, Sloan JA, Shanafelt TD. Single item measures of emotional exhaustion and depersonalization are useful for assessing burnout in medical professionals. J Gen Intern Med. 2009;24(12): 1318-1321.

19. Weidner AKH, Phillips RL Jr, Fang B, Peterson LE. Burnout and scope of practice in new family physicians. Ann Fam Med. 2018;16(3):200-205.

20. Maslach C, Jackson SE, Leiter MP. The Maslach Burnout Inventory (3rd ed.). In: Evaluating Stress: A Book of Resources.; 1996:191-218.

21. Houkes I, Winants $Y$, Twellaar M, Verdonk P. Development of burnout over time and the causal order of the three dimensions of burnout among male and female GPs. A three-wave panel study. BMC Public Health. 2011;11:240.

22. Lee RT, Ashforth BE. A meta-analytic examination of the correlates of the three dimensions of job burnout. J Appl Psychol. 1996;81(2): 123-133.

23. Shanafelt TD, Dyrbye LN, Sinsky C, et al. Relationship between clerical burden and characteristics of the electronic environment with physician burnout and professional satisfaction. Mayo Clin Proc. 2016;91(7):836-848.

24. Shanafelt TD, Mungo M, Schmitgen J, et al. Longitudinal study evaluating the association between physician burnout and changes in professional work effort. Mayo Clin Proc. 2016;91(4):422-431.

25. Shanafelt T, Sloan J, Satele D, Balch C. Why do surgeons consider leaving practice? J Am Coll Surg. 2011;212(3):421-422.

26. Dyrbye LN, Varkey P, Boone SL, Satele DV, Sloan JA, Shanafelt TD. Physician satisfaction and burnout at different career stages. Mayo Clin Proc. 2013;88(12):1358-1367..

27. Halbesleben JRB, Osburn HK, Mumford MD. Action research as a burnout intervention: reducing burnout in the federal fire service. J Appl Behav Sci. 2006;42(2):244-266..

28. Hansen A, Peterson LE, Fang B, Phillips RL Jr. Burnout in young family physicians: variation across states. J Am Board Fam Med. 2018;31(1):7-8..

29. Shanafelt TD, Balch CM, Bechamps G, et al. Burnout and medical errors among American surgeons. Ann Surg. 2010;251(6):995-1000.

30. Puffer JC, Knight HC, O'Neill TR, et al. Prevalence of burnout in board certified family physicians. J Am Board Fam Med. 2017;30(2): 125-126.

31. Helfrich CD, Simonetti JA, Clinton WL, et al. The association of team-specific workload and staffing with odds of burnout among VA primary care team members. J Gen Intern Med. 2017;32(7): $760-766$.

32. Willard-Grace R, Knox M, Huang B, Hammer H, Kivlahan C, Grumbach K. Burnout and health care workforce turnover. Ann Fam Med. 2019;17(1):36-41.

33. Sinsky CA, Willard-Grace R, Schutzbank AM, Sinsky TA, Margolius $D$, Bodenheimer $T$. In search of joy in practice: a report of 23 high-functioning primary care practices. Ann Fam Med. 2013;11(3):272-278

34. Mishra P, Kiang JC, Grant RW. Association of medical scribes in primary care with physician workflow and patient experience. JAMA Intern Med. 2018;178(11):1467-1472.

35. Nantsupawat A, Nantsupawat R, Kunaviktikul W, Turale S, Poghosyan L. Nurse burnout, nurse-reported quality of care, and patient outcomes in Thai hospitals. J Nurs Scholarsh. 2016;48(1):83-90.

36. Gómez-Urquiza JL, De la Fuente-Solana El, Albendín-García L, Vargas-Pecino C, Ortega-Campos EM, Cañadas-De la Fuente GA. Prevalence of burnout syndrome in emergency nurses: metaanalysis A. Crit Care Nurse. 2017;37(5):e1-e9.

37. Dyrbye LN, West CP, Shanafelt TD. Defining burnout as a dichotomous variable. J Gen Intern Med. 2009;24(3):440, author reply 441. 